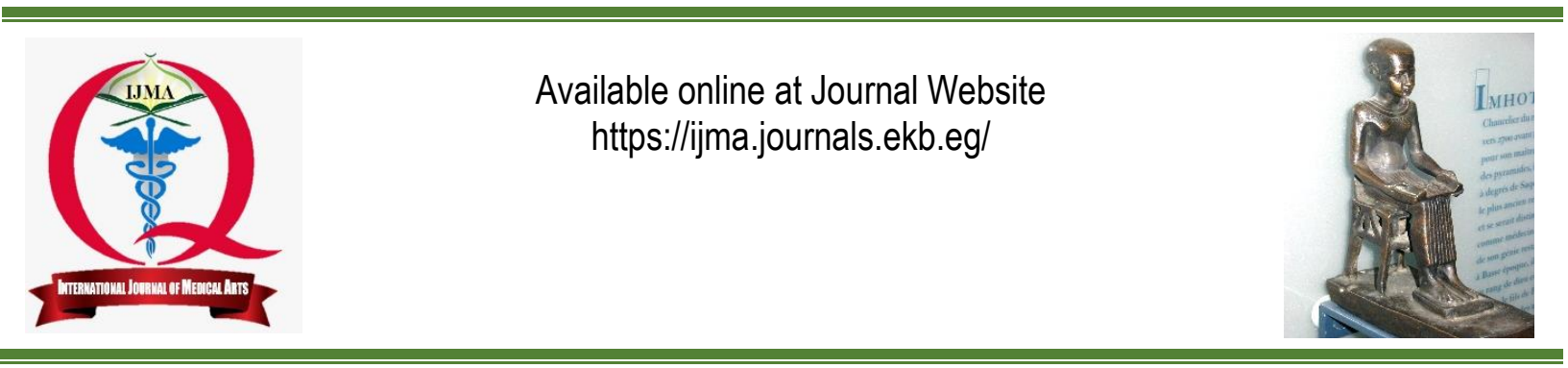

Original article

\title{
Prevalence of Intrauterine Adhesions after Hysteroscopic Myomectomy by Integrated Bigatti Shaver [IBS]
}

\author{
Eman Yahia Awad; Mohamed Anter; Waleed Ahmed Ayad \\ Department of Obstetrics and Gynecology, Damietta Faculty of Medicine, Al-Azhar University, Egypt. \\ Corresponding author: Eman Yahia Awad \\ Email: eman201095@gmail.com
}

Received at: June 13, 2019; Revised At: July 5, 2019.

Accepted at: September 27, 2019; Available online at: September 27, 2019

DOI: 10.21608/ijma.2019.13646.1015

\section{ABSTRACT}

Background: Myomectomy is the best procedure for treatment of uterine fibroids. However, post-myomectomy adhesions represent a major problem. The introduction of hysteroscopic myomectomy thought to reduce these adhesions. However, the incidence is not determined after different shavers.

Aim of the work: To assess the prevalence of adhesions in women undergoing IBS-myomectomy for uterine fibroids.

Patients and methods: The study included 70 women indicated for IBS myomectomy. Eligible females were submitted to full history taking, clinical examination and routine laboratory investigations. In addition, all patients underwent transvaginal ultrasonography. Fibroid grade was defined and hysteroscopic myomectomy. Then office hysteroscopy was done after 3 months of the procedure, and any pathology especially intrauterine adhesions was reported. Patients were classified into two groups; with adhesions and without adhesions.

Results: The incidence of adhesions was $5.7 \%$. The development of adhesion was usually associated with multiple myomas present in posterior position who need long resection time and usually the resection is incomplete. The adhesions were usually mild [75\%] and there was no complications were reported after hysteroscopic myomectomy.

Conclusion: The incidence of intrauterine adhesion after the hysteroscopic myomectomy by IBS is low

Keywords: Hysteroscopy; Myomectomy; Uterine adhesions; Synechiae; Integrated Bigatti Shaver.

This is an open access article under the Creative Commons license [CC BY] [https://creativecommons.org/licenses/by/2.0/]

Please cite this article as: Awad EY, Anter M, Ayad WA. Prevalence of Intrauterine Adhesions after Hysteroscopic Myomectomy by Integrated Bigatti Shaver [IBS]. IJMA 2019; 1[2]: 103-109. 


\section{INTRODUCTION}

Uterine fibroids have a significant impact on women's health. Most risk factors are not modifiable, including premenopausal age, age at menarche, and childbearing[1]. Fibroids are the mostcommon pelvic tumors in women of reproductive age ${ }^{[2]}$. They can cause abnormal uterine bleeding and their removal is beneficial in the treatment of heavy menstrual bleeding associated with fibroids for women who would like to preserve their uterus and fertility [3]. Other complications included pressure symptoms, increased urinary frequency, bowel disturbances, or pelvic pain ${ }^{[4]}$. The need to treat submucosal fibroids is widely accepted, but fibroids in other locations and sizes continue to present a clinical proplem [5].

Hysterectomy may be the first choice for women who have completed their child-birth; however, many women may prefer to keep the uterus if the uterine fibroids-related symptoms can be appropriately controlled[4]. Myomectomy is the most suitable surgical option for women who desire preservation of their fertility. Furthermore, the consequences of myomectomy on reproductive function have remained controversial[6].

Hysteroscopic myomectomy is the gold standard procedure for treatment of submucous fibroids. Hysteroscopic myomectomy techniques for removal of submucous fibroid still have controversies ${ }^{[7]}$. Bigatti et al.[8] developed an alternative prodedure for operative hysterectomy called IBS $®$ Integrated Bigatti Shaver that improve the visualization during the procedure, as the tissue chips are removed from the uterine cavity at the same time of removal, reduces several problems of conventional resectoscopy such as fluid overload and with a lower risk of uterine perforation.

\section{AIM OF THE WORK}

The present study aims to assess the prevalence of adhesions in women undergoing IBSmyomectomy for uterine fibroids.

\section{PATIENTS AND METHODS}

The present study is a retrospective follow up study. The study included 70 women indicated for IBS myomectomy. They were selected from Obstetrics and Gynecology Department. After full explanation of the study protocol for each patient, an informed consent was signed by each participant. In addition, the study protocol was approved by the research and local ethics committee. Patient was included if she fulfilled the following criteria: older than 18 years of age, had submucous myoma G0, G1, the size of myoma $\leq 3$ $\mathrm{cm}$. On the other side, patient was excluded if there was: suspicion of malignancy, active pelvic inflammatory disease, probability of pregnancy, severe vaginal infection, bleeding tendency, cardiac disease. Eligible females were submitted to full history taking, clinical examination and routine laboratory investigations. In addition, all patients underwent transvaginal ultrasonography. Fibroid grade was defined by the classification developed by Wamsteker et al.[9] and adopted by the European Society for Gynecological Endoscopy [ESGE], which considers only the degree of myometrial penetration of the submucous fibroid. According to this classification, a fibroid $G 0$ is completely within the uterine cavity and appears only jointed to the cavity wall by a thin pedicle; a fibroid G1 has its larger part [ $>50 \%]$ in the uterine cavity; $[9,10]$. Then office hysteroscopy was done after 3 months of the procedure, and any pathology especially intrauterine synechiae is reported and classified according to American fertility society. Patients were classified into two groups; with adhesions and without adhesions.

Statistical analysis: the collected data were organized, tabulated and analyzed using statistical package for social science [SPSS] version 20 $[I B M \otimes S P S S \otimes$, Chicaco, USA]. The numerical variables were expressed as mean and standard deviation [SD], while categorical variables were presented as number and percentages. Comparison between groups was done by independent samples student [t] test and chi square for qualitative variables. $P$ value $<0.05$ was considered significant.

\section{RESULTS}

The present study included 70 females who underwent hysteroscopic myomectomy by IBS $®$ shaver. Three months after hysteroscopy, adhesions were developed in 4 patients [5.7.0\%] [Table 1].

Patients" age ranged from 24 to 36 years; and there was non-significant decrease of age in females with adhesions when compared to those who had no adhesions [ $29.50 \pm 5.44$ vs $31.21 \pm 3.85$ years respectively]. The indication of myomectomy was infertility in $36 \%$, abnormal uterine bleeding [AUB] in $38 \%$ and pelvic pain in $26 \%$ and there was 
no significant difference between adhesion and non-adhesion groups.

The number of myoma in studied females was single in 45 females [64.3\%] and multiple in 25 females [35.7\%] and there was statistically significant increase of patients with multiple myomas in adhesion group when compared to nonadhesion group [ $50.0 \%$ vs $6.5 \%$ respectively]. The site of myoma was anterior in $44 \%$, posterior in $10 \%$ and fundal in $46 \%$ and there statistically significant ice of posterior position in group with adhesion when compared to non-adhesion group $[75.0 \%$ vs $4.3 \%$ respectively]. Myoma grade was G0 in $22 \%$, $\mathrm{G} 1$ in $20.0 \%$ and there was no significant difference between group with adhesions when compared to non-adhesion group [Table 2].

The myoma size ranged from 1.5 to 3 and there was no significant difference between adhesion and non-adhesion groups.

As regard to cervical dilatation time, it ranged from 1 to 3 minutes, while total operative time ranged from 24 to 38 minutes; and there was no significant difference between adhesion and non- adhesion groups. On the other hand, the resection time ranged from 14 to 23 minutes and there was statistically significant increase of resection time in adhesion when compared to non-adhesion groups [20.25 \pm 0.95 vs $17.65 \pm 1.82$ minutes respectively]. The amount of fluids used during hysteroscopy ranged from 2000 to $3750 \mathrm{cc}$, and there was no significant difference between adhesion and nonadhesion groups. Myoma removal was complete in $96 \%$ of all studied females and incomplete in $4 \%$. All cases in non-adhesion group had complete resection, while $50 \%$ of females with adhesion had complete resection. Thus, there was significant increase of incomplete resection in adhesion when compared to non-adhesion group $[50.0 \%$ vs $0.0 \%$ respectively]. Duration of hospital stay after the hysteroscopy, it ranged from 3 to 8 hours; and there was no significant difference between both adhesion and non-adhesion groups. The grade of adhesion was mild in 3 out of 4 females [75.0\%] and moderate in only one female [25.0\%] [Table 3]. No complications were reported in the present work.

Table [1]: Incidence of adhesions among studied patients

\begin{tabular}{|c|c|c|}
\hline & Frequency & Percent \\
\hline Adhesions & 4 & 5.7 \\
\hline No adhesions & 66 & 94.3 \\
\hline Total & 0 & 100.0 \\
\hline
\end{tabular}

Table [2]: The number of myomas among studied patients

\begin{tabular}{|c|c|c|c|c|c|c|c|c|}
\hline & \multicolumn{2}{|c|}{ With Adhesions [n=4] } & \multicolumn{2}{|c|}{ No Adhesions [ $n=46$ ] } & \multicolumn{2}{|c|}{ Total } & \multirow[t]{2}{*}{$P$ value } \\
\hline & & $\mathrm{N}$ & $\%$ & $\mathrm{~N}$ & $\%$ & $n$ & $\%$ & \\
\hline \multirow[t]{2}{*}{ Number } & Single & 2 & $50.0 \%$ & 43 & $93.5 \%$ & 45 & $64.3 \%$ & \multirow[t]{2}{*}{$0.005^{*}$} \\
\hline & Multiple & 2 & $50.0 \%$ & 23 & $6.5 \%$ & 25 & $35.7 \%$ & \\
\hline \multirow[t]{3}{*}{ Site } & Anterior & 1 & $25.0 \%$ & 26 & $39.4 \%$ & 72 & $38.6 \%$ & \multirow{3}{*}{$\begin{array}{c}P< \\
0.001^{*}\end{array}$} \\
\hline & Posterior & 3 & $75.0 \%$ & 7 & $10.6 \%$ & 10 & $14.3 \%$ & \\
\hline & Fundal & 0 & $0.0 \%$ & 33 & $50.0 \%$ & 33 & $47.1 \%$ & \\
\hline \multirow{2}{*}{$\begin{array}{l}\text { Myoma } \\
\text { Grade }\end{array}$} & G0 & 2 & $50.0 \%$ & 9 & $19.6 \%$ & 11 & $22.0 \%$ & \multirow[t]{2}{*}{0.29} \\
\hline & G1 & 2 & $50.0 \%$ & 9 & $19.6 \%$ & 10 & $20.0 \%$ & \\
\hline
\end{tabular}

Table [3]: Operative data among studied females

\begin{tabular}{|c|c|c|c|c|}
\hline & Adhesion [n=4] & No adhesions [n=66] & $P$ \\
\hline \multicolumn{2}{|c|}{ Cervical dilatation time [min] } & $1.25 \pm 0.50$ & $1.32 \pm 0.52$ & 0.77 \\
\hline \multicolumn{2}{|l|}{ Operative time [min] } & $34.25 \pm 5.566$ & $32.95 \pm 3.14$ & 0.46 \\
\hline \multicolumn{2}{|l|}{ Resection time [min] } & $20.25 \pm 0.95$ & $17.65 \pm 1.82$ & $0.008^{*}$ \\
\hline \multicolumn{2}{|l|}{ Amount of fluid } & $3062.50 \pm 773.92$ & $2940.21 \pm 391.52$ & 0.58 \\
\hline \multirow{2}{*}{$\begin{array}{l}\text { Myoma } \\
\text { Resection }\end{array}$} & Complete & $2[50.0 \%]$ & $46[70.0 \%]$ & \multirow[t]{2}{*}{$<0.001^{*}$} \\
\hline & Incomplete & $2[50.0 \%]$ & $23[30 . .0 \%]$ & \\
\hline \multicolumn{2}{|c|}{ Duration of hospital stay } & $5.25 \pm 1.89$ & $4.91 \pm 0.89$ & 0.51 \\
\hline \multirow[t]{2}{*}{ Grade of adhesion } & Mild & $3[75.0 \%]$ & & \\
\hline & Moderate & $1[25.0 \%]$ & & \\
\hline
\end{tabular}




\section{DISCUSSION}

The present study was designed to evaluate the prevalence of intra uterine adhesion after hysteronscopic myomectomy. It was carried out at the Department of Obstetrics and Gynecology. It included 70 females who underwent hysteroscopic myomectomy for different causes using the IBS ${ }^{2}$ integrated Bigatti Shaver, and reviewed for intrauterine adhesions 3 months after the primary procedure.

As regard to indication of myomectomy, it was infertility in 36\%, abnormal uterine bleeding [AUB] in $38 \%$ and pelvic pain in $26 \%$ and there was no significant difference between adhesion and nonadhesion groups. These results going in agreement with previous studies showed that, the most common clinical presentation symptoms of uterine myomas are bleeding, secondary anemia due to menometrorrhagia, metrorrhagia or intermenstrual bleeding, pain, bulk symptoms, sexual dysfunction, and infertility [11]. More than just one symptom is present in up to $62 \%$ of the affected woman[12].

In addition, Bhandari et al. [13] reported that, most of the participants [72.55\%] were cases of primary infertility.

In their study, Bahar et al. ${ }^{14]}$ reported that, the most prevalent symptoms were irregular uterine bleeding [35.5\%] and infertility [21.9\%]. About 20\% of women had no symptoms and underwent a hysteroscopic procedure because of an abnormal finding at imaging during the routine annual checkup. This is quietly different from the present work and can be explained by different inclusion criteria.

In addition, Yendru et al.[15] reported that, females with uterine fibroids presented with different symptoms, like abnormal uterine bleeding [AUB], infertility and recurrent pregnancy loss [RPL]

In the present study, there was no complications reported. Di Spezio Sardo et al.[16] reported that, the double flow monopolar or bipolar resectoscope is still considered the first choice instrument to perform major operative hysteroscopical and urological procedures. However, many problems, technique related, remain still unsolved. For example, in monopolar resectoscope, the absorption of large volumes of electrolyte free, low viscosity fluid such as sorbitol-mannitol may result in volume overload with water intoxication. Volume overload may cause pulmonary edema, and water intoxication may lead to hyponatremia, and cerebral edema. Also, the use of bipolar resectoscopy does not prevent from these complications. If the use of isotonic solutions such as $0.9 \%$ sodium chloride [as in the present work] prevents from dilution hyponatremia, the risk of fluid overload is still maintained. In addition, several case reports have shown that massive fluid absorption during hysteroscopic myomectomy with normal saline solution resulted in severe hyperchloremic metabolic acidosis and dilution coagulopathy resolved with diuretic therapy[17,18].

The high safety of IBS is explained by the following facts: it is able to remove the tissue chips at the same time as removal so that the procedure, always done under visual control, becomes faster, very precise, easier to perform, and with less complications ${ }^{[19] .}$

A randomized prospective study published by Bigatti et al. on Gynecological Surgery in February 2012 showed that especially for the treatment of large polyps and myomas up to $2 \mathrm{~cm}$ it has several well-described advantages ${ }^{[8]}$.

Emanuel et al.[20] reported that, the diameter of an intrauterine pathology is strongly related to the operation time and to the complication rate. Bigatti et al. ${ }^{[8]}$ showed that both operating and removal times were statistically significantly in favor of the IBS $®$. No bleeding or major complication was observed in the IBS $₫$ group. Not only operation time but also total fluid loss appeared to be better with the IBS $\otimes$ system. In fact, this study demonstrated that using the IBS $\otimes$ for myoma removal, the median total operating time was 23.25 min with a medium resection time of $15.08 \mathrm{~min}$. In addition, the main advantage of the IBS $\otimes$ was that the myomas were effectively enucleated from their fovea, and the intramural site of insertion of the myoma was removed. The surrounding healthy endometrium was avoided without any thermal injury occurring compared with the less precise behavior of conventional resectoscopy. No coagulation was needed, and there were no excess bleeding problems.

Yang et al. [21] showed that the intrauterine Bigatti shaver is a safe and effective method for resection of endometrial polyps. The continuous flow of fluid and suction of tissue fragments offers a definite advantage by providing constant clear 
visualization. This is crucial in hysteroscopic surgery as an obscured operating field is the main reason for serious complications such as uterine perforation and prolonged surgery leading to fluid overload.

No reports were found for post-IBS $₫$ uterine adhesion in literature. Here, the incidence of adhesion post-hysteroscopic myomectomies were reported to be $8 \%$ which is significantly lower than other shavers reported in the previous studies. For example, with conventional resectoscopy, the adhesion formation is one of the most common complications of hystero-scopic myomectomy reported up to $31.3 \%$ in a single myoma and $45.5 \%$ in multiple myoma as reported by Deans and Abbott [22].

Results of the present work, confirmed that, the post-hysteroscopic adhesions is associated with multiplicity of myomas, as there was statistically significant increase of patients with multiple myomas in adhesion group when compared to nonadhesion group [ $50.0 \%$ vs $6.5 \%$ respectively].

Shokeir et al.[23] reported a $60 \%$ rate of intrauterine adhesions with degree I, 2-4 weeks after resectoscopic procedures for different endocavitary pathologies. Even though the study considered all hysteroscopic procedures and no detailed information was available regarding the prevalence after myomectomy, only $25 \%$ of the studied population showed an anatomically uninjured uterine cavity. A later follow-up evaluation at 12 months showed a significant difference in distribution, with a prevalence of vascular adherences compared to filmy synechiae.

In addition, Touboul et al.[24] reported an incidence of $7 \%$ with bipolar resection, while resection with monopolar electrodes was reported to be $30-40 \%$. They attributed the low rate of synechiae to the use of bipolar energy, considering it safer, less prone to accidental electric fluxes, and therefore not damaging the healthy myometrium.

In many studies, the rate of intrauterine adhesions is variable from $1.7 \%$ to $78 \%$ depending on the use of electrocautery and adhesion preventive strategies ${ }^{[25,26]}$. The reported rate in the present work [5.7\%] lies within the reported rate in literature.
On the other hand, Mazzon et al.[27] reported that, in 29 patients [4.23\%], synechiae of the uterine cavity after resectoscopic surgery was found. This value is much lower than the present work, and it may be attributed to different inclusion criteria, where they included all myomas regardless of its size and included all females regardless of their age group.

As regard resection in the present work, it was complete in $96 \%$ of all studied females and incomplete in 4\%. All cases in non-adhesion group had complete resection, while $50 \%$ of females with adhesion had complete resection. Thus, there was significant increase of incomplete resection in adhesion when compared to non-adhesion group [50.0\% vs $0.0 \%$ respectively]. These results are comparable to previous works reported the effectiveness of bipolar resectoscope [irrespective of the different shaver].

Mazzon et al.[27] reported that, the persistence in the uterine cavity of myomas that were only partially removed during resectoscopic surgery [residue myoma] may play a role in the development of longterm adhesions. The traumatized residual portion of the myoma in contact with the endometrium of the opposite side may facilitate the formation of columnar fibrous adherences. Therefore, a followup evaluation after a resectoscopic procedure is important for evaluating the complete removal of a myoma with a new surgical treatment. In their study, during the follow-up phase, new myomas or residual myomas were detected in 56 patients [8.17\%] [12\% in the present study].

In the present work, there was no significant association between the size of fibroid and incidence of postoperative intrauterine adhesions. These results are comparable to those reported by Asgari et al.[28] who found a non-significant relation between the two.

The grade of adhesion in the present work was mild in 3 out of 4 females [75.0\%] and moderate in only one female [25.0\%].

Bhandari et al.[13] reported that, of the 50 patients underwent myomectomy, intrauterine adhesions were observed in consequent hysteroscopy in 11 cases [21.57\%]. They added, of these 11 adhesion cases, mild adhesions were noted in seven cases, while moderate adhesions 
were present in three cases. Severe type of adhesion was observed in only one patient. The higher rate of adhesion in their work can be explained by the fact that, they included all myoma types and sizes, and the presence of multiple myomas in their study.

In summary, the IBS $₫$ proves to be an effective and safe new instrument for the removal of myomas. It is smaller. In addition, surgery is not interrupted by tissue chips removal which will shorten the total operating time with increasing learning curve. It is also proposed that, electrical current-free resection of myomas could significantly reduce the postoperative adhesions formation. However, due to small number of patients included in the present study, future trials on wide range of patients are recommend to confirm this proposal.

\section{Conflict of Interest}

Authors declare that, there was no conflicts of interest.

\section{REFERENCES}

1. Wise LA, Laughlin-Tommaso SK. Uterine leiomyomata. In: Goldman MB, Troisi R, Rexrode KM, editors. Women and health. San Diego: Academic; 2013. p. 285-306.

2. Ordulu Z. Fibroids: Genotype and Phenotype. Clin Obstet Gynecol. 2016 Mar; 59[1]:25-9. [DOI: 10.1097/ GRF.0000000000000177].

3. Saridogan E. Surgical treatment of fibroids in heavy menstrual bleeding. Women's Health [Lond Engl] 2016; 12[1]:53-62. [DOI: 10.2217/whe.15.89].

4. Horng HC, Wen KC, Su WH, Chen CS, Wang PH. Review of myomectomy. Taiwan J Obstet Gynecol. 2012 Mar; 51[1]:7-11. [DOI: 10.1016/ j.tjog.2012.01.003].

5. Purohit P, Vigneswaran K. Fibroids and Infertility. Curr Obstet Gynecol Rep. 2016; 5: 81-88. [DOI:10.1007/s13669-016-0162-2].

6. Wong L, Brun JL. Myomectomy: technique and current indications. Minerva Ginecol. 2014 Feb; 66[1]:35-47. [PMID: 24569403].

7. Korkmazer E, Tekin B, Solak N. Ultrasound guidance during hysteroscopic myomectomy in G1 and G2 Submucous Myomas: for a safer one step surgery. Eur J Obstet Gynecol Reprod Biol. 2016 Aug; 203:108-11. [DOI: 10.1016/j.ejogrb. 2016.03.043].

8. Bigatti G, Ferrario C, Rosales M, Baglioni A, Bianchi S. IBS $尺$ Integrated Bigatti Shaver versus conventional bipolar resectoscope: a randomised comparative study. Gynecol Surg. 2012;9[1]:63-72. [DOI: 10.1007/s10397011-0701-9].

9. Wamsteker K, Emanuel MH, de Kruif JH. Transcervical hysteroscopic resection of submucous fibroids for abnormal uterine bleeding: results regarding the degree of intramural extension. Obstet Gynecol 1993; 82: 736-740. [PMID: 8414318].
10. Salim R, Lee C, Davies A, Jolaoso B, Ofuasia E, Jurkovic D. A comparative study of three-dimensional saline infusion sonohysterography and diagnostic hysteroscopy for the classification of submucous fibroids. Hum Reprod 2005; 20: 253-257. [DOI: 10.1093/ humrep/deh557].

11. Khan AT, Shehmar M, Gupta JK. Uterine fibroids: current perspectives. Int J Womens Health 2014; 6:95-114. [DOI: 10.2147/IJWH. S51083].

12. Sparic $\mathbf{R}$, Terzic $\mathbf{M}$, Tinelli $A$. Uterine fibroids-clinical presentation and complications. Acta Chir lugosl. 2014; 61[3]:41-8. [DOI: 10.2298/ ACl1403041S].

13. Bhandari S, Ganguly I, Agarwal P, Singh A, Gupta N. Effect of myomectomy on endometrial cavity: A prospective study of 51 cases. J Hum Reprod Sci 2016; 9:107-11.[DOI:10.4103/0974-1208.183509].

14. Bahar R, Shimonovitz M, Benshushan A, Shushan A. Case control study of complication associated with bipolar and monopolar hysteroscopic operations. J Min Invasive Gynecol 2013; 20:376-80. [DOI:10.1016/j.jmig. 2012.12.012].

15. Yendru KS, Yelamanchi SD, Vaddiraju GB. Hysteroscopic Resection of Submucous Fibroids in Symptomatic Women. J Obstet Gynaecol India. 2019 Apr;69[2]:166172. [DOI: 10.1007/s13224-018-1139-z].

16. Di Spezio Sardo A, Mazzon I, Bramante S, Nappi C. Hysteroscopic myomectomy: a comprehensive review of surgical techniques. Hum Reprod Update 2008; 14[2]:101-19. [DOI:10.1093/humupd/dmm041].

17. Shaafer M, Von Ungern-Sternberg BS, Wight $E$. Isotonic fluid absorption during hysteroscopy. Anesthesiology 2005; 103:203-204. [DOI: 10.1097/ 00000542200507000-00029].

18. Van Kruchten PM, Vermelis JM, Herold I. Hypotonic and isotonic fluid overload as a complication of hysteroscopic procedures: two case reports. Minerva Anestesiol 2010; 76[5]:373-7. [PMID: 20395900].

19. Bigatti G. The Shaver Technique for Operative Hysteroscopy. Chapter 55, In: A. Tinelli et al. [eds.], Hysteroscopy; Springer International Publishing AG 2018; pp 635-648.

20. Emanuel MH, Hart A, Wamsteker K, Lammes F. An analysis of fluid loss during transcervical resection of submucous myomas. Fertil Steril. 1997;68[5]:881-6. [DOI: 10.1016/s0015-0282 [97] 00335-x].

21. Yang L, Rajesh H, Yu S. The Intrauterine Bigatti Shaver for Endometrial Lesions: Experience and Modifications. Open J Obstet Gynecol 2017; 7: 1-6. [10.4236/ojog.2017.71001]

22. Deans R, Abbott J. Review of intrauterine adhesions. J Minim Invasive Gynecol 2010; 17 [5]: 555-69. [DOI:10.1016/j.jmig.2010.04.016].

23. Shokeir TA, Fawzy M, Tatongy $M$. The nature of intrauterine adhesions following reproductive hysteroscopic surgery as determined by early and late follow-up hysteroscopy: clinical implications. Arch Gynecol Obstet 2008; 277:423-7. [DOI: 10.1007/s00404007-0475-5]. 


\section{Awad et al.}

24. Touboul C, Fernandez H, Deffieux X, Berry R, Frydman $R$, Gervaise A. Uterine synechiae after bipolar hysteroscopic resection of submucosal myomas in patients with infertility. Fertil Steril 2009; 92:1690-1693. [DOl:10.1016/j.fertnstert.2008.08.108].

25. Gambadauro P, Gudmundsson J, Torrejón R. Intrauterine adhesions following conservative treatment of uterine fibroids. Obstet Gynecol Int 2012; 2012:853269. [DOI: 10.1155/2012/ 853269]

26. Taskin O, Sadik S, Onoglu A, Gokdeniz R, Erturan E, Burak $F$, Wheeler JM. Role of endometrial suppression on the frequency of intrauterine adhesions after resectoscopic surgery. J Am Assoc Gynecol Laparosc 2000; 7:351-4. [DOl: 10.1016/s1074-3804[05]60478-1].
27. Mazzon I, Favilli A, Cocco P, Grasso M, Horvath S, Bini V, Di Renzo GC, Gerli S. Does cold loop hysteroscopic myomectomy reduce intrauterine adhesions? A retrospective study. Fertil Steril 2014; 101: 294-98.e3.

28. Asgari Z, Hafizi L, Hosseini R, Javaheri A, Rastad H. Intrauterine synechiae after myomectomy; laparotomy versus laparoscopy: Non-randomized interventional trial. Iran J Reprod Med 2015; 13:161-8. [PMID: 26000007]. 